\title{
Comparison between different methods of adjustment of thyroxin replacement dose after total thyroidectomy for bengin thyroid disease
}

\author{
Ashraf Hegab, MD; Mohamed El-Serafy, MD; Hossam Salah, MD
}

Department of General Surgery, Ain Shams University, Cairo, Egypt.

\begin{abstract}
Introduction: Levothyroxin (T4) is commonly employed to correct thyroid hormone deficiency from various causes, and as total thyroidectomy is increasingly used to treat benign thyroid conditions, lifelong treatment with optimal dose of thyroxin (T4) is required which is difficult to predict. This study investigated factors that might predict the ideal T4 dose, and testing the body mass index as predictive factor with the aim of reducing delays in achieving normal thyroid function after surgery.

Methods: This study was conducted on 52 patients presented with benign thyroid disease and operated upon with total thyroidectomy. The patients were prospectively randomized into two comparable groups of patients. One group of patients was given the routinely used titration method of thyroid replacement therapy, while the other used an algorithm for T4 dosage based on patient weight (postoperative weight) which was subsequently applied in previous published literatures. Patient and operative variables that might predict time to achieve normal thyroid function and optimal T4 replacement dose were examined.

Results: The median time to achieve normal thyroid function was 16 (range 8-33) weeks in the first group in which the traditional titration method was used and large changes in T4 dose needed. But it was only 8 (range 4-17) weeks in the second group in which the weight algorithm was used. In multivariate analysis, the best predictor of optimal T4 dose was body weight. The use of a weight-related algorithm improved time to achieve normal thyroid function. There was a much stronger association between the L-T4 dosage and body mass.

Conclusion: The T4 replacement dosage after total thyroidectomy is largely influenced by body weight particularly in subjects with high BMI values. Use of a weight-related algorithm shortens the time required to attain a stable dose of L-T4 compared with use of standard T4 dose-titration methods.
\end{abstract}

Keywords: Thyroidectomy, Levothyroxine, Body weight, Benign thyroid disease.

\section{Introduction:}

Levothyroxin (L-T4) is commonly employed to correct thyroid hormone deficiency from various causes. ${ }^{1}$ Recently there has been an increasing tendency to replace traditional subtotal thyroidectomy which carries an appreciable risk of recurrence with total thyroidectomy in the treatment of benign thyroid disease. ${ }^{1,2}$ In the hands of experienced endocrine surgeons, this radical approach eliminates recurrence, yet can be achieved with morbidity rates at least as good as those following subtotal resection. ${ }^{3}$ After total thyroidectomy there is a requirement for lifelong thyroxin replacement therapy. ${ }^{3}$ Methods for monitoring and adjusting thyroid hormone replacement after surgery vary widely between surgical units, but most of them use a dose-titration method involving commencement of thyroxin (T4) at an initial empirical dose (commonly $100 \mu \mathrm{g}$ ) with subsequent dose adjustment after thyroid function tests and assessment of patient symptoms. ${ }^{4}$ However, this method can lead to long delays in achieving adequate replacement in some patients. ${ }^{4,5}$ Also, both excess and insufficient thyroid hormone produce adverse effects in various tissues, and careful 
monitoring is advisable to establish the optimal dose of L-T4. ${ }^{6}$ Beside clinical evaluation, the sensitive immunometric TSH assays currently available allow precise L-T4 dosage in most patients. ${ }^{7,8}$ Nevertheless, substantial interindividual variations of L-T4 requirements occur depending on the patient's age, gender, and body size, the presence of residual functioning thyroidal tissue, concurrent nonthyroidal diseases, pharmacological agents, or specific physiologic conditions, such as pregnancy, may also call for adjustment of the L-T4 daily dose. ${ }^{9-11}$ There is a general agreement in literature that the ideal body weight should be considered to calculate the amount of L -T4 to be administered in each patient, and evidence has been provided that the lean body mass is a predictor of the daily requirements for thyroid hormone. ${ }^{12}$ It has been demonstrated that thyroxin requirements are related to patient age and lean body mass, and that normal thyroid function can be achieved with thyroxin doses between 1 and $2.2 \mu \mathrm{g} / \mathrm{kg}$ body weight. ${ }^{10,12}$

The aim of this study was aiming to compare an algorithm for optimal thyroxine dosage based on the weight, with the standard dosetitration technique. Also, factors that may lead to a delay in achieving normal thyroid function after thyroidectomy for benign disease were examined.

\section{Patients and methods:}

This study was conducted at Ain Shams University Hospitals between January 2006 and April 2008 and involved 52 patients (36 women and 16 men; median age 36.6 years (range 22-54), who had undergone total thyroidectomy for uncontrolled Graves' disease or multinodular goiter (simple and toxic) with postoperative thyroxin used for replacement and normal thyroid function documented after surgery.

\section{Operative procedure:}

Under general endotracheal anesthesia, the patient is placed in a supine position with the neck extended. A low collar incision is made and carried down through the subcutaneous tissue and platysma muscle. Superior and inferior subplatysmal flaps are developed, and the strap muscles retracted laterally.
The thyroid lobe is bluntly dissected free from its investing fascia and rotated medially. The middle thyroid vein is ligated. The superior pole of the thyroid is dissected free, and care is taken to identify and preserve the external branch of the superior laryngeal nerve. The superior pole vessels are ligated adjacent to the thyroid lobe to prevent damage to this nerve.

The inferior thyroid artery and recurrent laryngeal nerve are identified. We do not ligate the inferior thyroid artery laterally as a single trunk; rather, its branches are ligated individually on the capsule of the lobe after they have supplied the parathyroid glands to preserve blood supply to the parathyroid glands. The parathyroid glands are identified, and an attempt is made to leave each with an adequate blood supply while moving the gland off the thyroid lobe. Also, care is taken to try to identify the recurrent laryngeal nerve along its course as total thyroidectomy was the planed surgery. The nerve is gently unroofed from surrounding tissue, with care taken to avoid trauma to it especially near the junction of the trachea with the larynx, where it is adjacent to the thyroid gland. Once the nerve and parathyroid glands have been identified and preserved, the thyroid lobe can be removed from its tracheal attachments by dividing the ligament of Berry. The contralateral thyroid lobe is removed in a similar manner. Careful hemostasis and visualization of all important anatomic structures are mandatory for success. A small suction catheter is inserted through a small stab wound; it is generally removed within 2448 hours.

When closing, we do not tightly approximate the strap muscles in the midline; this allows drainage of blood superficially and thus prevents a hematoma in the closed deep space. Furthermore, we obtain better cosmesis by not approximating the platysmal muscle. Rather, the dermis is approximated by interrupted 30 sutures, and the epithelial edges are approximated with a running subcuticular 30 absorbable suture. Sterile Steri-strips are then applied and left in place for about a week Figure(1).

Patients who had not achieved normal thyroid function were excluded. They were divided into two groups; both groups (26 
patients) were age, sex and pre-operative thyroid status matched. In the first group (Group I) of patients a traditional dose-titration method for thyroxin replacement was used. Thyroxin was commenced on the first day after surgery, usually at a 'standard' dose of $100 \mu \mathrm{g}$. Patients were seen in the outpatient clinic initially at 4 weeks and subsequently after another 4 weeks or according to clinical need, with thyroxin

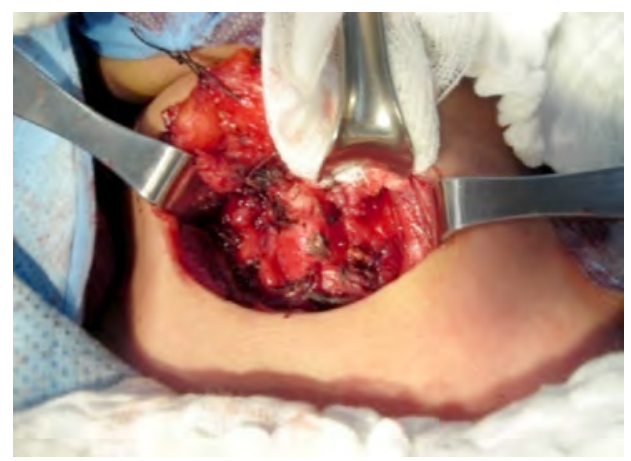

(A)

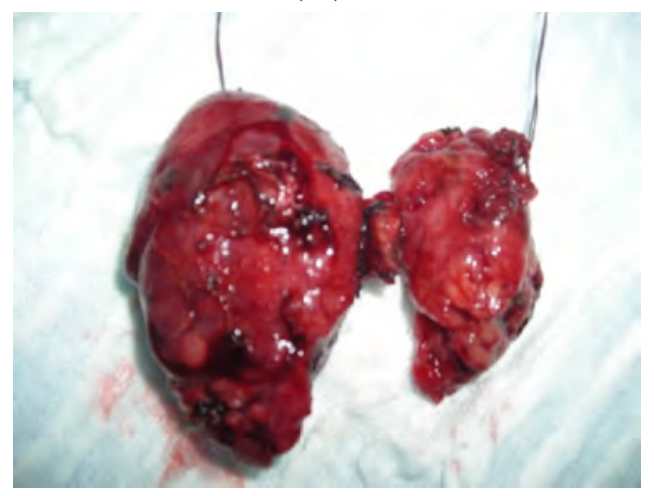

(C) dose titration in increments of $25-50 \mu \mathrm{g}$ depending on biochemical thyroid function tests results and patient symptoms. Thyroid hormone levels were assessed using a standard chemiluminescence assay. Study endpoints were the time taken to attain normal thyroid functions, defined as TSH level within the normal population range, and the dose of thyroxin needed to achieve this.

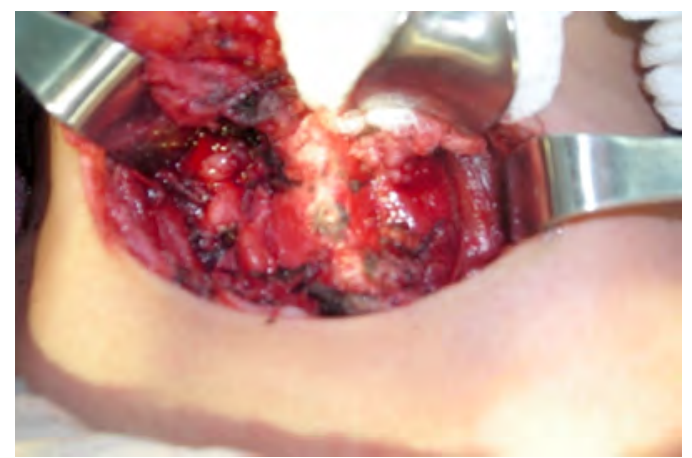

(B)

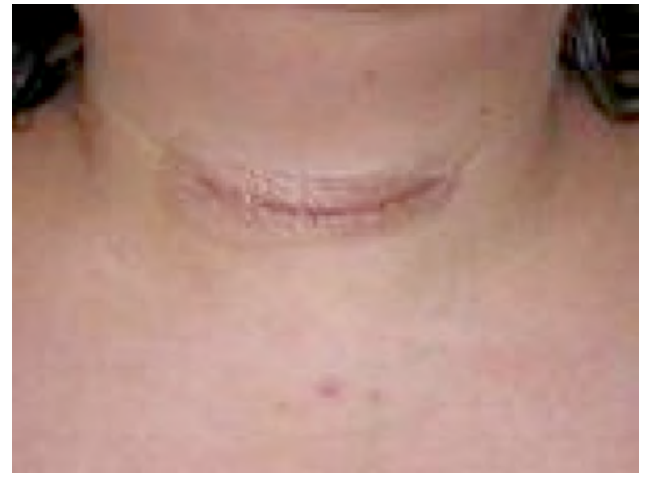

(D)

Figure (1): A,B) Operative field in female patient $38 y$ s presented with simple multinodular goiter during the operation for total thyroidectomy and showing excision of both thyroid lobes. C) Specimen of total thyroidectomy. D) Closure of the wound.

The time to achieve normal thyroid functions was defined as the interval between the date of surgery and the date of the first normal TSH measurement recorded in the patient's case notes. In patients who were thyrotoxic before operation, normal thyroid functions was deemed to have been achieved if free thyroid hormone levels were within the normal population range, even when TSH remained suppressed, as long as a subsequent normal TSH level was recorded without any intervening change in thyroxin dose. Variables examined in relation to the above endpoints were indication for surgery (Graves' disease versus multinodular goiter), preoperative thyroid status (toxic versus euthyroid), body weight (Kg), height (meter) and body mass index (BMI). While all other factors regarding patient age, sex, postoperative status between both groups are the same, also titration in group 2 is the same as group 1; as difference was only with the initial dosing between both groups). Because the literature published before suggested that lean body mass may be a better predictor of thyroxin dosage, ${ }^{7}$ the 'postoperative weight' was calculated for each patient [height (meter) ${ }^{2} \times 23$, where 23 is the 'ideal' BMI], as an estimate of lean body mass. Data of all these variables were completed for all patients. An algorithm for the dosage of thyroxin in relation to the body weight, used before in a study conducted by Olubowale and Chadwick, ${ }^{7}$ was subsequently applied to a consecutive series of 26 patients (Group II) who underwent total thyroidectomy for the similar benign indications Table(1). 
Table (1): Weight-related algorithm used in group II for calculation of postoperative T4 dose Olubowale and Chadwick, 2006.7

\begin{tabular}{|c|c|}
\hline $\begin{array}{c}\text { Patient Weight } \\
(\mathbf{k g})\end{array}$ & $\begin{array}{c}\text { T4 Dose } \\
(\boldsymbol{\mu g})\end{array}$ \\
\hline-53 & 100 \\
\hline $54-86$ & 125 \\
\hline $87-108$ & 150 \\
\hline$>108$ & 175 \\
\hline
\end{tabular}

Fasting serum samples were collected from all patients and processed within 30 minutes, after which they were kept frozen at $-20^{\circ} \mathrm{C}$. We assayed levels of free tri-iodothyronine (T3), free T4 by using T3, T4 Accubind ELISA Microwells, respectively [Monobind, Inc. Lake Forest, CA (92630) USA]. Values between 1.4 and $4.2 \mathrm{ng} / \mathrm{dl}, 0.8$ and $2.5 \mathrm{ng} / \mathrm{dl}$, were considered normal respectively. Serum TSH was assessed by using TSH, Accubind ELISA Microwells, [Monobind, Inc. Costa Mesa, CA (92627) USA]. Values between 0.28 and 5.6 $\mathrm{mU} / \mathrm{L}$ for TSH was considered normal.

\section{Statistical Analysis:}

Data were collected, revised, verified then edited on PC. Also, data were analyzed statistically using SPSS (statistical package for social sciences version 13). Statistical analysis was carried out to examine variables affecting thyroxin dosage using the student's$t$ test of independent variables. Data were expressed as mean $\pm \mathrm{SD}$, range and median. (The results were considered to be statistically significant at $\mathrm{P}$ value $<0.05$, highly significant at $P$ value $<0.001$ and insignificant at $P$ value $>0.05)$.

\section{Results:}

The mean age of the study group was 36.6 (range 22-54) years. Group I involved 26 patients 6 males (23\%) and 20 females (77\%). It also involved 8 toxic cases $(30.7 \%)$ and 18 euthyroid cases $(69.2 \%)$. While in Group II; it involved 26 patients 9 males $(34.6 \%)$ and 17 females $(65.3 \%)$ and also involved 10 toxic cases $(38.4 \%)$ and 16 euthyroid cases $(61.5 \%)$. The mean thyroxin dose for all the patients was $1.88 \mu \mathrm{g} / \mathrm{kg}$ body weight (range 1.5-2.3). In the first group of patients in whom the traditional titration method was used, the time taken to achieve normal thyroid function was highly variable with a median time of 16 (range 8-33) weeks, whereas in the second group of patients in whom the algorithm was used, this time was significantly reduced to 8 (range 417) weeks Figure(2).

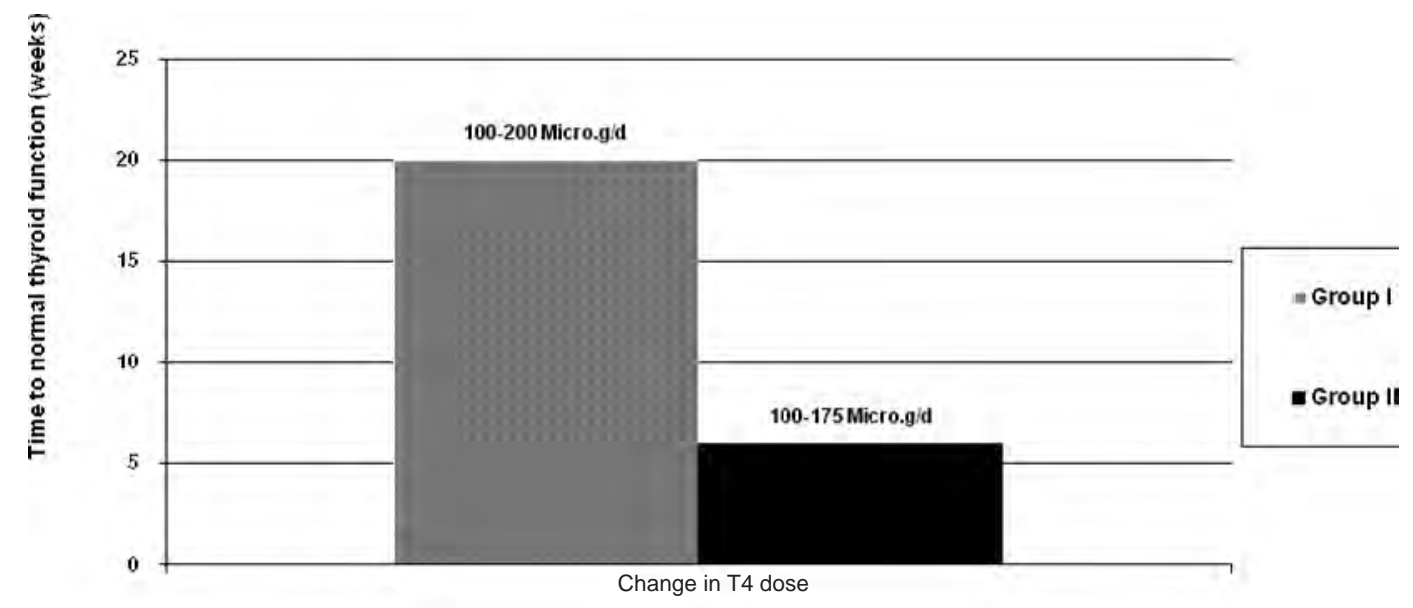

Figure (2): Time to achieve normal thyroid function in relation to change in thyroxin doses. 
Of the 26 patients in the first group who were initially started on $100 \mu \mathrm{g}$ per day only 4 patients $(15.3 \%)$ did not require further adjustment of the dose after 8 weeks, however, 17 patients $(65.3 \%)$ required further dose increments, and were given $50 \mu \mathrm{g}$ increments over the following 4 weeks which were grossly increased by time to achieve normal thyroid function. In Group I, 5 cases (19.2\%) required even more increments and doses of thyroxin increased up to $200 \mu \mathrm{g}$ per day over 24 weeks to achieve normal thyroid function. The weightrelated algorithm for calculation of postoperative T4 dose used for group II (in $25-\mu \mathrm{g}$ intervals, for simplicity of dosages) is shown in Table(1). The cohort of patients treated after introduction of the algorithm was well matched with the earlier group with respect to baseline characteristics, particularly the bodyweight Table(2).

Statistical analysis revealed that T4 dose required to achieve normal thyroid functions was not influenced by the pre-operative thyroid function status, or by the gender of the patient. But the body weight proved to be the best predictor of the required T4 dose Figure(3).

Table (2): Anthropometric measures, L-T4 daily dose, and serum hormonal concentrations in the two groups of patients (26 patients/ group).

\begin{tabular}{|c|c|c|}
\hline & $\begin{array}{c}\text { Group I } \\
\text { (26 patients/ group) }\end{array}$ & $\begin{array}{c}\text { Group II } \\
\text { (26 patients/group) }\end{array}$ \\
\hline $\begin{array}{l}\text { *Weight (kg) (postoperative) } \\
\text { *Height }(\mathrm{cm}) \\
\text { *BMI }(\mathrm{kg} / \mathrm{m} 2) \text { (postoperative) } \\
\text { *Age }(\mathrm{yr}) \\
\text { *Gender: } \\
\text {-Male } \\
\text {-Female } \\
\text { *Indication for surgery: } \\
\text { 1) Graves' disease. } \\
\text { 2) Multinodular goiter: } \\
\text { - Simple. } \\
\text { - Toxic. } \\
\text { 3) Thyroiditis. } \\
\text { *L-T4 dose ( } \mu \mathrm{g} / \mathrm{d}) \\
\text { *L-T4 dose }(\mu \mathrm{g} / \mathrm{kg} / \mathrm{d}) \\
\text { *Time passed to normal } \\
\text { thyroid function (weeks) }\end{array}$ & $\begin{array}{c}68 \pm 7.5 \\
166 \pm 8 \\
26 \pm 3.7 \\
39 \pm 11 \\
\\
6(23 \%) \\
20(77 \%) \\
6(23 \%) \\
16(61.5 \%) \\
2(7.6 \%) \\
2(7.6 \%) \\
150 \pm 25 \\
2.10 \pm 0.31 \\
16(8-33)\end{array}$ & $\begin{array}{c}74 \pm 10.0 \\
167 \pm 10.0 \\
29.9 \pm 5.6 \\
41 \pm 12 \\
9(34.6 \%) \\
17(65.3 \%) \\
8(30.7 \%) \\
15(57.7 \%) \\
2(7.6 \%) \\
1(3.8 \%) \\
125 \pm 25 \\
1.60 \pm 0.31 \\
8(4-17)\end{array}$ \\
\hline
\end{tabular}

Data are reported as median \pm range. To convert fT4 and fT3 to picograms per milliliter, multiply by 0.777 and 0.651 , respectively.

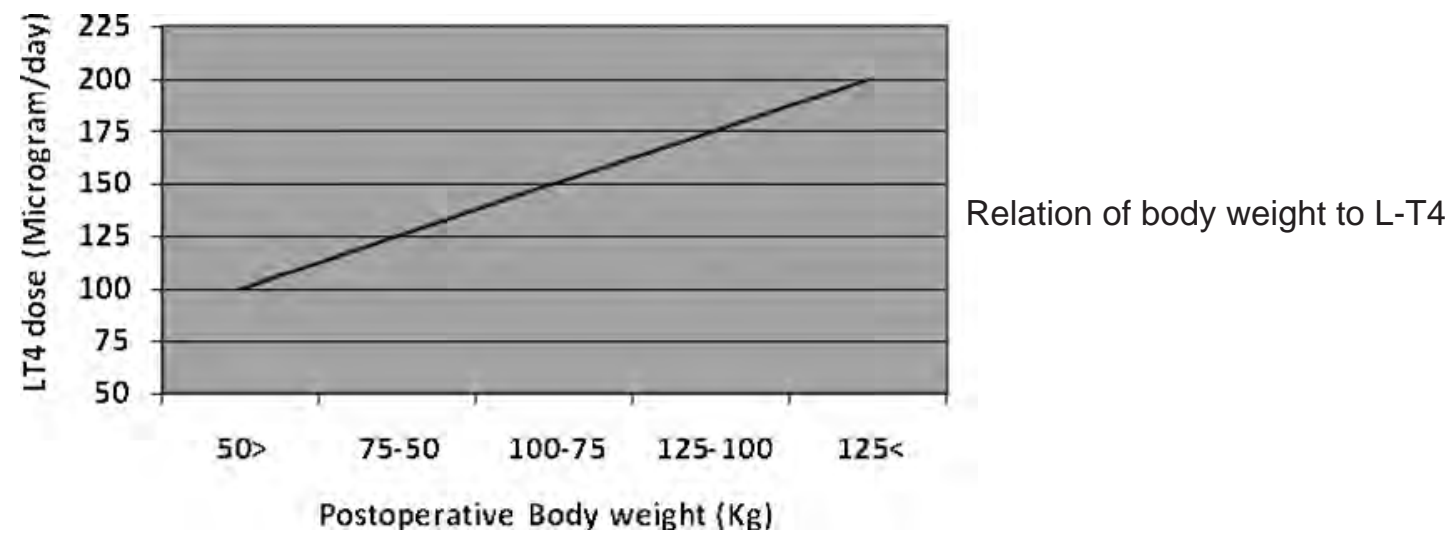

Figure (3): Correlations between the daily dose of L-T4 and total body weight in 52 total thyroidectomized patients on L-T4 therapy. 


\section{Discussion:}

Thyroxin replacement therapy is instituted to restore thyroid function to normal after total thyroidectomy for benign thyroid disease. It is important to achieve this promptly because over or under-replacement results in troublesome symptoms and in Graves ' disease may be associated with worsening of ophthalmopathy. ${ }^{2,3}$ A stable dose of L-T4 in treatment of thyroid diseases is achieved when the amount of hormone entering the blood stream equals the proportion that is metabolized, allowing constant serum concentrations of fT4, fT3, and TSH. ${ }^{4}$ When administered in the fasting state, about $80 \%$ of L-T4 contained in modern tablets is absorbed. After ingestion, serum T4 values peak at 2-4 $\mathrm{h}$ and return toward basal levels after about 6 h. 4,5 The serum half-life of T4 approximates 7 days in the euthyroid state, and its clearance depends mainly on deiodination and to a lesser extent on other pathways, such as sulfation and glucuronidation. ${ }^{6}$ The daily replacement dose of L-T4 to normalize serum TSH in adult hypothyroid subjects is on average, $1.6 \mu \mathrm{g} / \mathrm{kg}$ body weight. ${ }^{6}$ The amount of hormone to be administered must be increased when suppressed values of serum TSH are desired. In both cases, however, individual adjustments are usually required to attain the optimal daily dose. Although, the total daily requirements of L-T4 are related to body mass, unexplained difference can occur among individuals for the same age and body size, even in the absence of functioning thyroid tissue. 6,7

This study demonstrated that standard T4 dose-titration methods may result in prolonged delays in achieving normal thyroid function, particularly when the final, optimal dose of T4 is much greater than the dose commenced immediately after surgery. This is because titration of the T4 dose is usually undertaken in small increments of 25-50 $\mu \mathrm{g}$ (most of patients respond with $50 \mu \mathrm{g}$ ), with an interval of several weeks before thyroid function tests can meaningfully be repeated. Reducing these delays can be achieved only by determining which factors influence optimal $\mathrm{T} 4$ dose for each individual.

Much of the previous literature on $\mathrm{T} 4$ requirements has focused on primary hypothyroidism and these studies may not accurately predict $\mathrm{T} 4$ requirements after total thyroidectomy. The present results nonetheless confirm previous findings of a relationship between T4 dose and bodyweight. Moreover, the finding of a negative correlation between T4 dose expressed with respect to bodyweight and weight itself was also confirmed in the present report. Thus, lighter patients required proportionally greater doses of $\mathrm{T} 4$ per unit bodyweight than heavier patients, although the latter patients required higher absolute $\mathrm{T} 4$ doses. 7,8

Optimal weight-adjusted doses of T4 in this study were higher (median $1.88 \mu \mathrm{g} / \mathrm{kg}$ ) than baseline doses used in many surgical units (100 $\mu \mathrm{g}$ or $1.3 \mu \mathrm{g} / \mathrm{kg}$ ), ${ }^{3}$ and were greater than expected particularly in leaner patients. A T4 dose algorithm based on body weight, such as that in the present study, is therefore recommended, but an initial empirical dose of $150 \mu \mathrm{g}$ T4 for obese patients might be preferred if a standard dose-titration technique is used.9-11

This study also demonstrated that there is a large inter-individual variation in optimal T4 dose, only part of which can be explained by body weight. Patient compliance with replacement therapy may be an important factor in this respect. Although the principal determinant of delay to normal thyroid function is the need for large changes in T4 dose during follow-up after surgery, some of the longest delays nonetheless occurred in patients whose change in T4 dose was small, implying irregular compliance during this time interval. This proves the need for this algorithm as patients may not come for follow up with subclinical cases of hypo or hyperthyroidism and this may lead to a delay in the time needed for optimal correction. 


\section{References:}

1- Reeve TS, Delbridge L, Cohen A and Crummer P: Total thyroidectomy: The preferred option for multinodular goiter. Ann Surg 1987; 206: 782-786.

2- Russell CFJ: Management of benign nonendemic goiter. In: Textbook of endocrine surgery. Clark OH, Duh Q-Y (Editors); Philadelphia: WB Saunders (Publisher); 1997; p.22-31.

3- Miccoli P, Antonelli A, Iacconi P, et al: Prospective, randomized double-blind study about effectiveness of levothyroxine suppressive therapy in prevention of recurrence after operation: Result at the third year of follow-up. Surgery 1993; 114: 1097-1101.

4- Topliss DJ and Eastman CJ: Diagnosis and management of hyperthyroidism and hypothyroidism. Med J Aust 2004; 180: 186-193.

5- Wenzel KW: Optimization of levothyroxine treatment: Dosage dependence on the existing parenchymal mass, age, body weight and fasting intake. Dutsch Med Wochenschr 1986; 111: 1356-1362.

6- Banovac K, Carrington SA, Levis S et al: Determination of replacement and suppressive doses of thyroxin. J Int Med Res 1990; 18: 210-218.
7- Olubowale O, Chadwick DR: Optimization of thyroxin replacement therapy after total or near-total thyroidectomy for benign thyroid disease. British Journal of Surgery 2006; 93: 57-60.

8- Zbar AP, O'Higgins NJ: Use and abuse of thyroid stimulating hormone suppressive therapy in patients with nodular goiter and benign or malignant thyroid neoplasm. In: Textbook of endocrine surgery. Clark $\mathrm{OH}$, Duh Q-Y (Editors); Philadelphia: WB Saunders (Publisher); 1997; p.54-68.

9- Weetman AP: Thyroid-associated eye disease: Pathophysiology. Lancet 1991; 338: 25-28.

10-Rezzonico JN, Pusiol E, Saravi FD, et al: Management of overt and subclinical hypothyroidism: Factors influencing Lthyroxine dosage. Medicina (B Aires) 1999; 59: 698-704.

11-Elberling TV, Rasmussen AK, FeldtRasmussen, et al: Quality of life is decreased in patients with hyperthyroidism caused by Graves' disease. Eur J Endocrinol 2004; 151: 549-555.

12-Santini F, Pinchera A, Marsili A, et al: Lean body mass is a more important determinant of thyroxine dose than fat mass, age or sex. $J$ Clin Endocrinal Metab 2005; 90:124127. 